\title{
The galaxy cluster X-ray luminosity-gravitational mass relation in the light of the WMAP 3rd year data
}

\author{
T. H. Reiprich
}

\begin{abstract}
Argelander Institute for Astronomy (AIfA) ${ }^{\star}$, Bonn University, Auf dem Hügel 71, 53121 Bonn, Germany e-mail: thomas@reiprich.net
\end{abstract}

Received 29 April 2006 / Accepted 18 May 2006

\begin{abstract}
Context. The 3rd year WMAP results mark a shift in best fit values of cosmological parameters compared to the 1st year data and the concordance cosmological model.

Aims. We test the consistency of the new results with previous constraints on cosmological parameters from the HIFLUGCS galaxy cluster sample and the impact of this shift on the X-ray luminosity-gravitational mass relation.

Methods. The measured X-ray luminosity function combined with the observed luminosity-mass relation are compared to mass functions predicted for given cosmological parameter values.

Results. The luminosity function and luminosity-mass relation derived previously from HIFLUGCS are in perfect agreement with mass functions predicted using the best fit parameter values from the 3rd year WMAP data $\left(\Omega_{\mathrm{m}}=0.238, \sigma_{8}=0.74\right)$ and inconsistent with the concordance cosmological model $\left(\Omega_{\mathrm{m}}=0.3, \sigma_{8}=0.9\right)$, assuming a flat Universe. Trying to force consistency with the concordance model requires artificially decreasing the normalization of the luminosity-mass relation by a factor of 2 .

Conclusions. The shift in best fit values for $\Omega_{\mathrm{m}}$ and $\sigma_{8}$ has a significant impact on predictions of cluster abundances. The new WMAP results are now in perfect agreement with previous results on the $\Omega_{\mathrm{m}}-\sigma_{8}$ relation determined from the mass function of HIFLUGCS clusters and other X-ray cluster samples (the "low cluster normalization"). We conclude that - unless the true values of $\Omega_{\mathrm{m}}$ and $\sigma_{8}$ differ significantly from the 3rd year WMAP results - the luminosity-mass relation is well described by their previous determination from X-ray observations of clusters, with a conservative upper limit on the bias factor of 1.5. These conclusions are currently being tested directly in a complete follow-up program of all HIFLUGCS clusters with Chandra and XMM-Newton.
\end{abstract}

Key words. cosmological parameters - cosmic microwave background - dark matter - X-rays: galaxies: clusters galaxies: clusters: general

\section{Introduction}

The abundance of local galaxy clusters is very sensitive to the matter density, $\Omega_{\mathrm{m}}$, and the amplitude of density fluctuations, expressed as $\sigma_{8}$, in the Universe. This has long been known and the method has been applied often to constrain cosmological parameters (e.g., Henry \& Arnaud 1991; Bahcall \& Cen 1992 , 1993; Pierpaoli et al. 2003; Voevodkin \& Vikhlinin 2004). In practice, this test requires detailed knowledge about the number density of clusters and their gravitational mass. The number density as a function of, e.g., X-ray luminosity can be measured relatively easily while the accurate determination of the cluster mass is difficult and subject to systematic errors. For instance, mass determinations based on galaxy velocity dispersion and X-ray emission of the intracluster gas may be affected if the cluster is undergoing a merger event (e.g., Randall et al. 2002; Reiprich et al. 2004), gravitational lensing masses may be affected by projection effects (e.g., Clowe et al. 2004). In the era of precision cosmology and with the realization of very tight constraints on cosmological parameters especially from cosmic microwave background measurements (e.g., Spergel et al. 2003), these difficulties have lead to the idea to turn the argument around and assume the cosmological model to be known precisely and use

* Founded by merging of the Institut für Astrophysik und Extraterrestrische Forschung, the Sternwarte, and the Radioastronomisches Institut der Universität Bonn. the abundance of clusters to study cluster physics. For example, the X-ray luminosity-gravitational mass $\left(L_{\mathrm{X}}-M_{\text {tot }}\right)$ relation may be derived by combining the measured X-ray luminosity function with the assumed cluster mass function.

In the last years, a broad consensus has developed that we live in a concordance Universe $\left(\Omega_{\mathrm{m}}=0.3, \Omega_{\Lambda}=0.7, \sigma_{8}=0.9\right)$, based on consistent measurements from a multitude of completely independent observations (from cosmic microwave background, distant supernovae type Ia, large scale structure, galaxy clusters, etc., e.g., Bahcall et al. 1999; Wang et al. 2002; Turner 2002a,b); although the $\sigma_{8}$ constraint remained somewhat controversial (e.g., Wang et al. 2003). Then, the case was further strengthend when the 1st year results from WMAP returned more evidence for a flat Universe for reasonable values of the Hubble constant, and best fit values that are very close to the concordance values $\left(\Omega_{\mathrm{m}}=0.29, \sigma_{8}=0.9\right.$, Spergel et al. 2003, their Table 2).

Although the best fit $\Omega_{\mathrm{m}}$ and $\sigma_{8}$ values changed only within the uncertainties between the WMAP 1st year and 3rd year data, this has nonetheless dramatic consequences for predictions of cluster abundances, since these are very sensitive to even small changes in these values. This is illustrated in Fig. 1 where the cluster mass functions for the concordance cosmology and the new best fit values $\left(\Omega_{\mathrm{m}}=0.238, \sigma_{8}=0.74\right.$, Spergel et al. 2006; from now on referred to as "new cosmology") are compared. Also shown are data points from the HIFLUGCS mass 


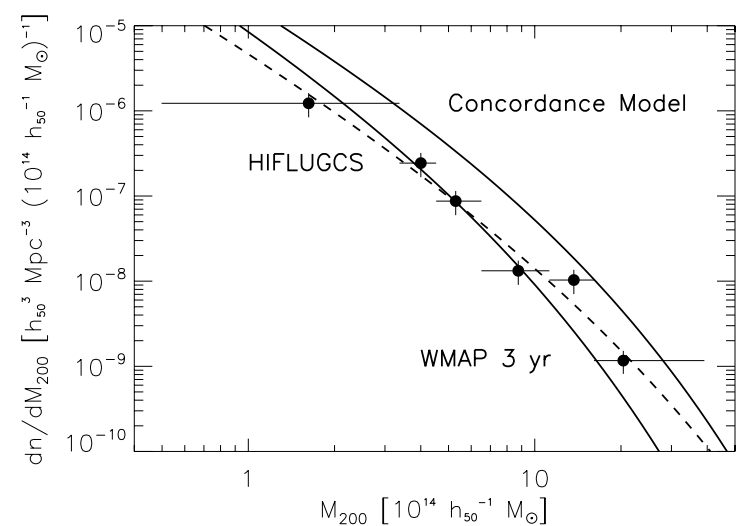

Fig. 1. Galaxy cluster mass functions. Filled circles: HIFLUGCS data (63 clusters); dashed line: best fit to HIFLUGCS data; upper solid line: predicted by concordance model $\left(\Omega_{\mathrm{m}}=0.3, \sigma_{8}=0.9\right)$, which was a very good fit to the WMAP 1st year data; lower solid line: predicted by best fit to the WMAP 3 rd year data $\left(\Omega_{\mathrm{m}}=0.234, \sigma_{8}=0.74\right)$. The concordance model predicts too many $(>200)$ clusters in the survey volume while the new cosmology predicts about the correct number. Adapted from Reiprich (2005, Fig. 10.87).

function, determined with individual mass measurements for 63 galaxy clusters, assuming the intracluster gas to be in hydrostatic equilibrium with the gravitational potential (Reiprich \& Böhringer 2002). The concordance model predicts more than 200 galaxy clusters in the survey volume while the new cosmology predicts $\sim 50$ clusters. It is to be expected that this change in best fit cosmology has significant consequences when deriving the cluster $L_{\mathrm{X}}-M_{\mathrm{tot}}$ relation by combining the measured luminosity function with the predicted mass function. The aim of this Letter is to illustrate these consequences and suggest a current best estimate of the $L_{\mathrm{X}}-M_{\mathrm{tot}}$ relation.

\section{Measuring and predicting the cluster $L_{\mathrm{x}}-M_{\text {tot }}$ relation}

The observed cluster $L_{\mathrm{X}}-M_{\text {tot }}$ relation was first studied in detail by Reiprich \& Böhringer (2002, RB02) using the X-ray flux-limited HIFLUGCS sample. It is difficult to accurately simulate the X-ray luminosity of galaxy clusters (e.g. Frenk et al. 1999) but it is reassuring that high resolution cosmological hydrodynamical simulations that include cooling and non-gravitational heating are able to reproduce this observed $L_{\mathrm{X}}-M_{\text {tot }}$ relation (e.g., Tornatore et al. 2003).

Since the scatter in this relation is finite, RB02 (Sect. 4.2), as well as Reiprich (2001, Sect. 6.4) and Ikebe et al. (2002, Sect. 2.3) mention explicitly that the normalization might be slightly biased high because, in a flux-limited sample, clusters with higher luminosity for a given mass have a higher likelihood to be included in the sample. In a recent detailed study, Stanek et al. (2006) quantify this bias by predicting the $L_{\mathrm{X}}-M_{\text {tot }}$ relation using cluster number counts and the scatter in the luminosity-temperature relation, assuming the cluster mass function in a concordance Universe $\left(\Omega_{\mathrm{m}}=0.3, \Omega_{\Lambda}=0.7\right.$, $\left.\sigma_{8}=0.9\right)$. They find the normalization measured by RB02 is biased high by a factor of $2-$ a significant bias.

The mass function measured with HIFLUGCS is not consistent with a concordance Universe (Fig. 1). RB02 found the relation $\sigma_{8}=0.43 \Omega_{\mathrm{m}}^{-0.38}$, which surprisingly implied $\sigma_{8}=0.68$ for a fiducial $\Omega_{\mathrm{m}}=0.3$, and discussed this "low cluster normalization" for the first time in detail. This relation is shown

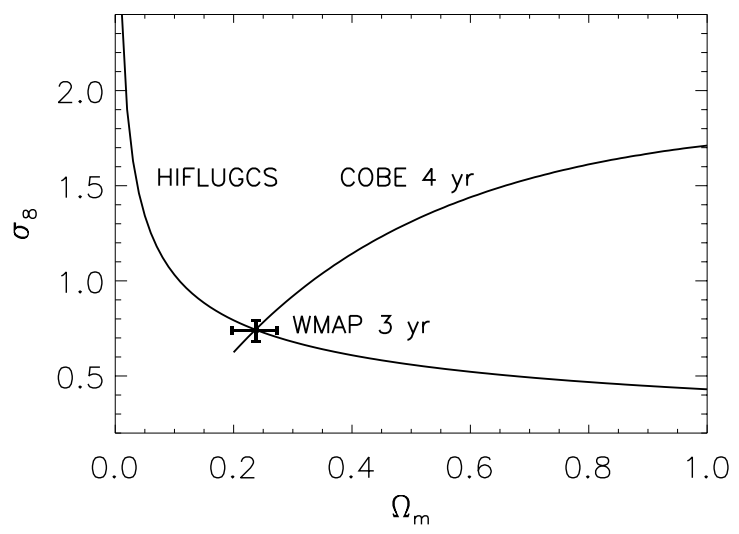

Fig. 2. The best fit model to the WMAP 3rd year data lies exactly at the intersection of the $\Omega_{\mathrm{m}}-\sigma_{8}$ relations from HIFLUGCS and COBE. COBE relation from Bunn \& White (1997), calculated using a code provided by T. Kitayama. Adapted from Reiprich (2003).

in Fig. 2. Also shown are the results from the COBE 4 year data (Bunn \& White 1997) and the WMAP 3rd year best fit value. The new WMAP result lies exactly at the intersection of the COBE and HIFLUGCS results. The fact that the WMAP value lies exactly on the HIFLUGCS curve also implies that the new cosmology predicts the correct cluster abundance. This suggests that the normalization of the $L_{\mathrm{X}}-M_{\text {tot }}$ relation measured for the HIFLUGCS clusters may actually not be biased very strongly.

To test this quantitatively, we determined "quasi-mass functions" from the HIFLUGCS luminosity function. In detail, the availability of individually measured masses for all HIFLUGCS clusters was ignored and the mass for each cluster was instead estimated from its luminosity and the $L_{\mathrm{X}}-M_{\text {tot }}$ relation $(M \mid L$ in Table 8 of RB02). With these "masses", the quasimass function was constructed. Then, predicted mass functions were fit to the quasi-mass function, following the procedure described in detail in RB02, resulting in best fit values ${ }^{1} \Omega_{\mathrm{m}}=0.22$ and $\sigma_{8}=0.74-$ almost precisely the values favored by the new cosmology (Fig. 3). Since Stanek et al. (2006) argue that the normalization of the HIFLUGCS $L_{\mathrm{X}}-M_{\mathrm{tot}}$ relation is overestimated by a factor of 2 , we divided the normalization by 2 and repeated the above procedure. The new best fit values were $\Omega_{\mathrm{m}}=0.29$ and $\sigma_{8}=0.88$; i.e., almost exactly the concordance cosmology assumed by Stanek et al. (2006). Therefore, assuming the new cosmology from the WMAP 3rd year data, we conclude that the factor of 2 bias found by Stanek et al. (2006) is due to assuming the "wrong" cosmological model. This conclusion is qualitatively consistent with their relation $L_{15,0} \propto \sigma_{8}^{-4}$ (note that the "In" in the relation they give in their abstract and in their derivation in Sect. 4.2 seems to be a typo), which implies a factor of 3 reduction in normalization when going from $\sigma_{8}=0.68$ to $\sigma_{8}=0.9$. Note that in a revised version of their paper, Stanek et al. will offer a "compromise" solution, assuming $\sigma_{8}=0.85$ (for $\Omega_{\mathrm{m}}=0.24$ ), resulting in moderate scatter in the $L_{\mathrm{X}}-M_{\mathrm{tot}}$ relation and a smaller bias (A. Evrard, priv. comm.).

The absence of a strong bias found here also implies that the intrinsic scatter in the $L_{\mathrm{X}}-M_{\text {tot }}$ relation may indeed be relatively small. Assume, unrealistically, that there is zero intrinsic

\footnotetext{
1 Note that Ikebe et al. (2002) and RB02 demonstrated that even for a local cluster sample; i.e., without information on cluster abundance evolution, the degeneracy between $\Omega_{\mathrm{m}}$ and $\sigma_{8}$ can be broken through the shape of the mass function (the resulting direct best fit values from the RB02 mass function are consistent within the statistical uncertainties with the new cosmology).
} 


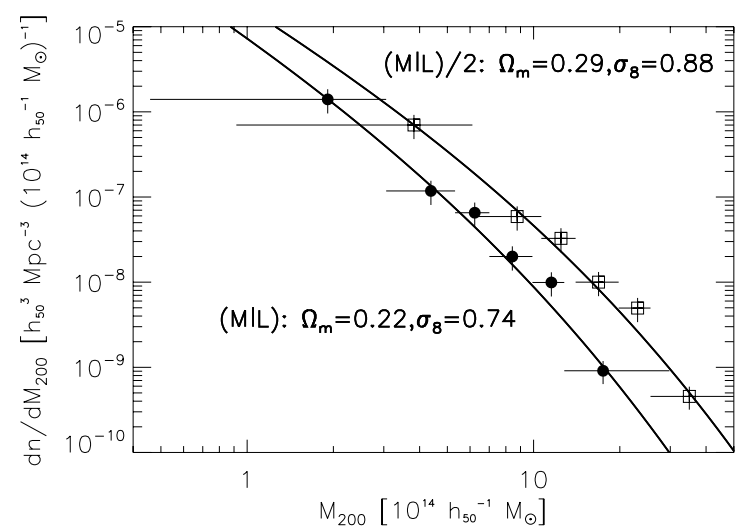

Fig. 3. Quasi-mass functions estimated from luminosity function and luminosity-mass relations. Filled circles: $L_{\mathrm{X}}-M_{\text {tot }}$ relation as measured with HIFLUGCS; open squares: normalization of this relation artificially divided by a factor of 2 . The solid lines indicate the respective best fit mass functions: $\left(\Omega_{\mathrm{m}}=0.22, \sigma_{8}=0.74\right)$ and $\left(\Omega_{\mathrm{m}}=0.29, \sigma_{8}=0.88\right)$; the former result is very close to the new cosmology favored by WMAP and the latter very close to the concordance model.

scatter. This would imply a bias factor of 1 (i.e., no bias at all) for cluster samples drawn from a given mass distribution, regardless of how the sample was selected, because all clusters with a given mass have exactly the same luminosity. The larger the scatter the larger the bias in flux-limited samples, therefore, a small bias indicates small scatter. Zhang et al. (2006) use 14 clusters from an almost volume-complete sample. As mentioned in Reiprich (2001), in truly volume-limited samples there should be no bias in the normalization of the $L_{\mathrm{X}}-M_{\text {tot }}$ relation, regardless of the amount of intrinsic scatter. In their XMM-Newton analysis Zhang et al. confirm normalization and scatter of the RB02 luminosity-mass relation, corroborating our result here that the bias is small.

Note that we do not argue here that the bias factor in the normalization of the $L_{\mathrm{X}}-M_{\mathrm{tot}}$ relation estimated from flux-limited samples is exactly 1 (see our references above), but rather that a bias factor of 2 as found by Stanek et al. (2006) is a strong overestimate unless the new WMAP results are "wrong" or unless another competing systematic effect conspires to hide a bias this large. That the intrinsic scatter is indeed larger than zero is, e.g., indicated by the observed segregation of cooling core clusters towards the high luminosity side for a given mass in the $L_{\mathrm{X}}-M_{\mathrm{tot}}$ relation (Y. Chen et al., in prep.; Reiprich \& Sarazin 2003) and by temporary luminosity increases in major mergers predicted by simulations (e.g., Randall et al. 2002). We are currently studying these effects in more detail with better data.

To derive an upper limit on the bias allowed by the WMAP 3 rd year data we used the largest $\Omega_{\mathrm{m}}$ and $\sigma_{8}$ values consistent with the quoted uncertainties: $\Omega_{\mathrm{m}}=0.27$ and $\sigma_{8}=0.79$ (Spergel et al. 2006, note that we combined the uncertainties in $\Omega_{\mathrm{m}} h^{2}$ and $h$ in a worst case fashion, to be conservative). Then the quasi-mass function fit procedure was repeated for varying bias factors. The largest bias factor for which the $1-\sigma$ error ellipse is still consistent with $\Omega_{\mathrm{m}}=0.27$ and $\sigma_{8}=0.79$ is 1.5 . Therefore, we conclude that the bias factor $\leq 1.5$, unless $\Omega_{\mathrm{m}}$ and/or $\sigma_{8}$ are larger than allowed by the WMAP 3rd year data. Without being less conservative, a tighter constraint on the bias parameter seems currently not feasible with this method because of the existing uncertainties in $\Omega_{\mathrm{m}}$ and $\sigma_{8}$ and the strong sensitivity of the cluster mass function on these parameters.

Despite the very good agreement with the new WMAP results, it should be noted that in their ROSAT and ASCA analysis
RB02 had to use rather simplifying assumptions in their determination of cluster masses. For instance, the intracluster medium was assumed to be isothermal throughout the entire cluster volume and for some clusters only a relatively small number of photons was available for the determination of the gas density profile. With the new generation of X-ray satellites these limitations can be overcome. Therefore, we are currently followingup all HIFLUGCS clusters with Chandra (Hudson et al., in prep.; Reiprich et al. 2006) and XMM-Newton (Nenestyan et al., in prep.). This should allow us to get a realistic estimate of the intrinsic scatter directly.

\section{Summary of conclusions}

1. The shift in best fit values for $\Omega_{\mathrm{m}}$ and $\sigma_{8}$ between WMAP 1st year and WMAP 3rd year data has a significant effect on predictions of cluster abundances because they are very sensitive to even small changes in these parameters.

2. The WMAP 3rd year data are now consistent with the low cluster normalization found previously.

3. The observed cluster X-ray luminosity-gravitational mass relation and X-ray luminosity function are in perfect agreement with mass functions predicted for $\Omega_{\mathrm{m}}=0.238$ and $\sigma_{8}=0.74$, as favored by the WMAP 3rd year data.

4. Assuming $\Omega_{\mathrm{m}}=0.238$ and $\sigma_{8}=0.74$, we find that the expected bias in normalization when determining the cluster luminosity-mass relation from flux-limited samples is small - much smaller than the factor of 2 claimed previously.

5. Assuming $\Omega_{\mathrm{m}}=0.27$ and $\sigma_{8}=0.79$, the largest values allowed by the new WMAP data, we derive a conservative upper limit on the bias factor of 1.5.

6. A small bias indicates that the intrinsic scatter in the luminosity-mass relation is small.

The RB02 results, together with results from other works (e.g., Borgani et al. 2001; Seljak 2002; Viana et al. 2002) that yielded the low cluster normalization, triggered a $\sigma_{8}$-debate, which seems to have resulted in a loss of trust in cosmological constraints derived from clusters within the cosmology community. Now that other well-respected methods like those that rely on measurements of anisotropies in the cosmic microwave background also tend to find lower values of $\sigma_{8}$, we hope that trust in clusters will be restored. Clusters are ideally suited to address the new fundamental questions in cosmology (e.g., Mohr 2005): planned X-ray cluster surveys like those to be performed with eROSITA ${ }^{2}$ are expected to yield up to 100000 clusters out to large redshifts. The resulting detailed measurements of the evolution of cosmic structure are among the most promising routes to pin down the nature and evolution of dark energy.

Acknowledgements. We thank the anonymous referee for quick and useful comments. T.H.R. acknowledges support by the Deutsche Forschungsgemeinschaft through Emmy Noether Research Grant RE 1462.

\section{References}

Bahcall, N. A., \& Cen, R. 1992, ApJ, 398, L81

Bahcall, N. A., \& Cen, R. 1993, ApJ, 407, L49

Bahcall, N. A., Ostriker, J. P., Perlmutter, S., \& Steinhardt, P. J. 1999, Science, 284, 1481

Borgani, S., Rosati, P., Tozzi, P., et al. 2001, ApJ, 561, 13

Bunn, E. F., \& White, M. 1997, ApJ, 480, 6

Clowe, D., De Lucia, G., \& King, L. 2004, MNRAS, 350, 1038

\footnotetext{
2 See, for instance,

http ://www .mpe.mpg . de/erosita/MDD-6.pdf
} 
Frenk, C. S., White, S. D. M., Bode, P., et al. 1999, ApJ, 525, 554

Henry, J. P., \& Arnaud, K. A. 1991, ApJ, 372, 410

Ikebe, Y., Reiprich, T. H., Böhringer, H., Tanaka, Y., \& Kitayama, T. 2002, A\&A, 383,773

Mohr, J. J. 2005, in Observing Dark Energy, ed. S. C. Wolff, \& T. R. Lauer, ASP Conf. Ser., 339, 140

Pierpaoli, E., Borgani, S., Scott, D., \& White, M. 2003, MNRAS, 342, 163

Randall, S. W., Sarazin, C. L., \& Ricker, P. M. 2002, ApJ, 577, 579

Reiprich, T. H. 2001, Ph.D. Thesis, Ludwig-Maximilians-Universität München [arXiv: astro-ph/0308137]

Reiprich, T. H. 2003, in Matter and Energy in Clusters of Galaxies, ed. S. Bowyer, \& C.-Y. Hwang (San Francisco: ASP Conf. Series), 301, 563 [arXiv:astro-ph/0207071]

Reiprich, T. H. 2005, in Introduction to Galactic and Extragalactic X-Ray Astronomy, ed. J. Kerp, \& T. H. Reiprich, http://www .astro.uni-bonn.de/ reiprich/lectures/ SS05/vorl 2005-07-07 extragal.pdf

Reiprich, T. H., \& Böhringer, H. 2002, ApJ, 567, 716

Reiprich, T. H., \& Sarazin, C. L. 2003, in The Riddle of Cooling Flows in Galaxies and Clusters of Galaxies, ed. T. H. Reiprich, J. C. Kempner, \& $\mathrm{N}$. Soker (electronic proceedings available at http: //www . astro.virginia.edu/coolflow)
Reiprich, T. H., Sarazin, C. L., Kempner, J. C. \& Tittley, E. 2004, ApJ, 608, 179 Reiprich, T. H., Hudson, D. S., Erben, T., \& Sarazin, C. L. 2006, in Relativistic Astrophysics and Cosmology - Einstein's Legacy, ed. B. Aschenbach, V. Burwitz, G. Hasinger, \& B. Leibundgut (Berlin, Germany: ESO Astrophysics Symposia, Springer Verlag) [arXiv: astro-ph/0603129] Seljak, U. 2002, MNRAS, 337, 769

Spergel, D. N., Bean, R., Dore', O., et al. 2006, ApJ, submitted [arXiv: astro-ph/0603449]

Spergel, D. N., Verde, L., Peiris, H. V., et al. 2003, ApJS, 148, 175

Stanek, R., Evrard, A. E., Böhringer, H., Schuecker, P., \& Nord, B. 2006, ApJ, submitted [arXiv:astro-ph/0602324]

Tornatore, L., Borgani, S., Springel, V., et al. 2003, MNRAS, 342, 1025

Turner, M. S. 2002a, ApJ, 576, L101

Turner, M. S. 2002b, Int. J. Mod. Phys. A, 17, 180

Viana, P. T. P., Nichol, R. C., \& Liddle, A. R. 2002, ApJ, 569, L75

Voevodkin, A., \& Vikhlinin, A. 2004, ApJ, 601, 610

Wang, X., Tegmark, M., Jain, B., \& Zaldarriaga, M. 2003, Phys. Rev. D, 68, 123001

Wang, X., Tegmark, M., \& Zaldarriaga, M. 2002, Phys. Rev. D, 65, 123001

Zhang, Y.-Y., Böhringer, H., Finoguenov, A., et al. 2006, A\&A, accepted [arXiv: astro-ph/0603275] 